\title{
Evidence for Social Learning in a Family Living Lizard
}

\section{OPEN ACCESS}

Edited by:

François Criscuolo,

Centre National de la Recherche Scientifique (CNRS), France

Reviewed by:

Thierry Bernard,

Centre National de la Recherche Scientifique (CNRS), France Anindita Bhadra, Indian Institute of Science Education and Research Kolkata, India

${ }^{*}$ Correspondence: Martin J. Whiting martin.whiting@mq.edu.au orcid.org/0000-0002-4662-0227

${ }^{\dagger}$ Fonti Kar orcid.org/0000-0002-2760-3974 Julia L. Riley orcid.org/0000-0001-7691-6910 Richard W. Byrne orcid.org/0000-0001-9862-9373 Daniel W. A. Noble orcid.org/0000-0001-9460-8743

¥These authors have contributed equally to this work.

Specialty section:

This article was submitted to Behavioral and Evolutionary Ecology,

a section of the journal

Frontiers in Ecology and Evolution

Received: 21 December 2017 Accepted: 08 May 2018 Published: 29 May 2018

Citation: Whiting MJ, Xu F, Kar F, Riley JL, Byrne RW and Noble DWA (2018) Evidence for Social Learning in a Family Living Lizard. Front. Ecol. Evol. 6:70. doi: 10.3389/fevo.2018.00070

\author{
Martin J. Whiting ${ }^{1 * t}$, Feng $X u^{1,2 \ddagger}$, Fonti Kar ${ }^{1,3+\neq}$, Julia L. Riley ${ }^{1,3 t}$, Richard W. Byrne ${ }^{4 \dagger}$ and \\ Daniel W. A. Noble ${ }^{1,3+}$
}

${ }^{1}$ Department of Biological Sciences, Macquarie University, Sydney, NSW, Australia, ${ }^{2}$ Key Laboratory of Biogeography and Bioresources in Arid Land, Xinjiang Institute of Ecology and Biogeography, Chinese Academy of Sciences, Ürümqi, China, ${ }^{3}$ Evolution \& Ecology Research Centre, School of Biological, Earth and Environmental Sciences, University of New South Wales, Kensington, NSW, Australia, ${ }^{4}$ School of Psychology and Neuroscience, University of St Andrews, St Andrews, United Kingdom

Social learning is widespread among family living species, particularly mammals and birds with relatively high levels of social complexity and overt social interaction. However, the occurrence of social learning has never been documented in lizards with kin-based sociality, which have less obvious social interactions. We tested for social learning in Australian tree skinks (Egernia striolata), a species that commonly lives in family groups in the wild, using a two-step foraging task. Lizards were randomly allocated to either a social learning treatment or a control group and presented first with an instrumental task requiring the displacement of a lid, followed by an association task, consisting of two dishes with different colored lids. Prior to each task, lizards in the social learning treatment observed a trained demonstrator extract a food reward while the control also viewed a conspecific, but in the absence of the foraging task. The social learning treatment and control group solved the instrumental task at similar rates, but in the association task lizards in the social learning treatment made fewer errors and reached our learning criterion sooner. To the best of our knowledge, we present the first evidence for social learning in a lizard with kin-based sociality.

Keywords: social learning, lizard, sociality, Egernia, cognition

\section{INTRODUCTION}

Social learning-the process whereby an individual acquires new information through the observation of, or interaction with, another individual or its products (Hoppitt and Laland, 2013) has been documented in a wide range of organisms including insects (Leadbeater and Chittka, 2007; Alem et al., 2016), fishes (Helfman and Schultz, 1984; Reader et al., 2003; Thonhauser et al., 2013), lizards (Noble et al., 2014; Kis et al., 2015), turtles (Wilkinson et al., 2010; Davis and Burghardt, 2011), birds (Lefebvre, 1986; Holzhaider et al., 2010), and mammals (Reader and Laland, 2002; Thornton and Malapert, 2009; Thornton and Clutton-Brock, 2011; Whiten, 2011). Acquiring key information through social learning is thought to be adaptive because it is a short-cut to learning, particularly when individual learning is costly (e.g., foraging time, exposure to predators, energetic costs, etc.; Hoppitt and Laland, 2013). However, using social information is not always cost-free (Giraldeau et al., 2002).

Information obtained socially may not always be accurate and consequently some individuals may forego social information in favor of trial-and-error learning. Theoretical models predict that 
social learning only translates into fitness benefits when copying is rare (Boyd and Richerson, 1985; Giraldeau et al., 2002). If the frequency of social learners is high and fewer individuals are sampling the environment, then social learning will become less reliable. This effect is further compounded by dynamic environments where it will become less likely that any one individual will have accurate information about the environment. Under these circumstances, social learning will be a conditional strategy (Enquist et al., 2007). Alternatively, an individual may always copy first, and then revert to individual learning only when social learning is too costly or unprofitable (i.e., a criticalsocial-learner strategy; Enquist et al., 2007).

Social learning can be constrained by an individual's social environment and age. For example, adult male eastern water skinks (Eulamprus quoyii) do not use social information to solve a foraging task whereas sub-adult males do (Noble et al., 2014). Social dominance may also influence the acquisition of social information (Hoppitt and Laland, 2013). In some bird species that forage in small groups, a significant difference in competitive ability (i.e., social status) predicts whether individuals use social information (Barta and Giraldeau, 1998; Giraldeau and Beauchamp, 1999; Liker and Bartas, 2002). Under strong dominance asymmetry, dominant individuals are more likely to act as "scroungers" (i.e., observers) while subordinates are "producers" (i.e., inadvertent demonstrators) that use individual learning to locate food patches (Barta and Giraldeau, 1998). Likewise, when competitive asymmetries are less pronounced, differences in strategy begin to fall away (Barta and Giraldeau, 1998; Liker and Bartas, 2002). Therefore, whether individuals use social information is dependent on a range of ecological and social factors, and requires explicit testing.

Primates and birds have long been the banner organisms for social learning because of the often socially complex societies that they live in (Whiten, 2000; Byrne, 2015). In particular, social learning occurs disproportionally among kin (Laland, 2004). For example, a meta-analysis of social learning revealed a high frequency of social learning between mothers and their offspring among non-human primates (Reader, 2000). Social learning among kin is likely to be common because kin share genes and a common environment-interacting more frequently as a result, and therefore experiencing similar selective pressures. More importantly, social information is likely to be reliable because of kin selection: it pays to help kin, or to at least not deceive them, because of the fitness benefits derived from relatedness (Hamilton, 1964; Laland, 2004; Hoppitt and Laland, 2013). Honey bee waggle dances are perhaps one of the best examples of social learning among kin (Leadbeater and Chittka, 2007). Female workers share up to three-quarters of their genes and therefore, have high fitness benefits from imparting information about the location of nectar to colony mates (Laland, 2004).

Many family living species have been the focus of social learning studies because they have parental care and a high degree of social interaction (Laland, 2004). However, some organisms, such as lizards, live in stable family groups in the absence of overt parental care and their social interactions may be less pronounced. For example, species in the Australian Egernia group of skinks commonly live in nuclear families and may have several generations of offspring living together (Chapple, 2003; While et al., 2015; Whiting and While, 2017). The parental unit is monogamous and offspring gain protection, particularly from infanticide, by associating with their parents (O'Connor and Shine, 2004). These stable family groups are predicted to use social information because of kin selection and the fact that they share a common environment where selective pressures are similar among group members. The Australian tree skink (Egernia striolata) commonly lives facultatively in family groups in the wild (Duckett et al., 2012). The only test of social learning in this family-living species of lizard was for juvenile tree skinks (E. striolata) raised under two social treatments (isolated or in pairs). This study found no evidence of social learning between juveniles and an unrelated, unfamiliar adult demonstrator (Riley et al., 2018). However, the use of social information can depend on many factors like age, the relationship between demonstrator and observer, and rearing environment (Barta and Giraldeau, 1998; Noble et al., 2014). In this study, we tested for social learning in the same species (E. striolata) using wild-caught adult female lizards. We could not directly test for familial effects on social learning because we were unable to establish the relatedness of individuals used in this study. We opted to use only females because of the greater likelihood of social feedback in the case of males. For example, males of some lizard species may influence the occurrence of learning because of social feedback in a dominant-subordinate relationship (Noble et al., 2014; Kar et al., 2017). We hypothesized that females use social information and predicted that individuals with access to taskspecific information (via a demonstrator) would make fewer errors than control females with no such access to information about how to solve a task.

\section{MATERIALS AND METHODS \\ Overview}

We tested for social learning using 56 adult female E. striolata divided equally between demonstrators and observer lizards, in two batches. Because they were wild-caught as adults, we could not estimate their exact age. Lizards were captured by noosing or manipulated out of crevices and captured by hand near Albury, New South Wales (35.98'S, 146.97'E) and, before this study, maintained in all-female circular outdoor enclosures $3-\mathrm{m}$ in diameter while being used in a separate study. They were fed crickets or baby food 3 days/week prior to trials, and had access to wild insects. During social learning trials, we paired lizards in opaque plastic tubs $(690[\mathrm{~L}] \times 470$ [W] $\times$ $455[\mathrm{H}] \mathrm{mm}$ ) separated in half by a fixed transparent sheet of Perspex ${ }^{\circledR}$ and a removable opaque wooden divider. This allowed us to keep lizards physically separated, while also controlling when they viewed each other. Each lizard had a small plastic refuge, a water bowl and the substrate of the enclosure was newspaper. We conducted two trials a day, in the morning (09:00-11:30 h) and the afternoon (13:30-16:00 h), with a $2 \mathrm{~h}$ interval between trials. All trials were remotely filmed using CCTV cameras (Digital Video Recorder, model no. H. 264) and the researcher left the room immediately following the setup of the task. For consistency, a single individual (FX) scored all 
videos. Lizards remained in the same enclosure for the duration of the experiment.

\section{Social Demonstration Protocol}

We followed the same basic protocol as Noble et al. (2014), Kar et al. (2017), and Riley et al. (2018). We first trained all lizards to eat baby food (Heinz ${ }^{\circledR}$, fruity pear flavor) from an open, opaque dish (diameter: $54 \mathrm{~mm}$, height: $14 \mathrm{~mm}$ ) mounted on a small wooden block (Figure 1). We covered the sides of a clear dish in black tape to prevent the lizard from seeing its contents. We considered lizards to be trained if they ate food from the dish in 5/6 trials. At the onset of a trial, we removed the opaque divider and the observer lizard's refuge and water bowl to provide an unobstructed view of the demonstrating lizard. After $1 \mathrm{~h}$ of viewing, the opaque divider was replaced to separate lizards and give the observer lizard the opportunity to perform the task. Observer lizards were allocated to either a social learning treatment $(n=15)$ or control group $(n=13)$. In the social learning treatment, the observer lizard viewed the demonstrator access the food reward by displacing a lid from the rewardcontaining dish, while the control observed another lizard in the absence of any dishes. In the case of trials with two dishes, the lid was fixed to the incorrect dish, thereby preventing the demonstrator from removing it. As a consequence, the observer always received reliable information because it only saw the demonstrator displace the correct lid.

\section{Instrumental Task}

We first tested for social learning in an instrumental task by presenting lizards with a single dish covered by a yellow lid. We trimmed the lid's lip to ensure lizards could displace it without too

\section{Trained demonstrator Socially-learning observer}

\section{(a) Task 1: Instrumental task}
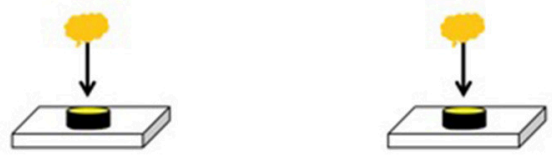

(b) Task 2: Association task
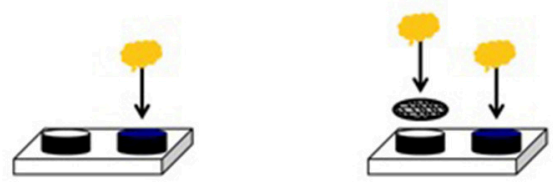

FIGURE 1 | The apparatus on the left was presented to trained demonstrators while the apparatus on the right was presented to the social learning observers. (a) In the instrumental task lizards were required to remove a yellow lid to access a food reward from a covered well. (b) In the association task the food reward was under the blue lid (correct choice) but inaccessible under the white lid (incorrect choice) much manipulation, in order to access the food reward. Observer lizards were required to displace an opaque yellow lid from the dish to get the food reward (Figure 1a). When lizards successfully displaced the lid in 5/6 trials, they were considered to have learnt this task. For each trial, we recorded if they successfully removed the lid and the latency (seconds) to accessing the food reward. Two lizards did not meet our learning criterion but were trained to displace the lid before moving to the next task. We used a 5/6 learning criterion in this task because it is a motor task that requires lizards to remove the lid from a single dish. Once a lizard removes the lid for the first time, they do so again in all subsequent trials making this a robust criterion. This instrumental task primarily equips lizards for the next task, which is a more robust test of social learning.

\section{Association Task}

We tested for social learning in an associative learning task by presenting observer lizards with a choice of two dishes covered by differently colored lids, only one of which contained a food reward (Figure 1b). The reward-containing, correct dish, was covered with a blue lid, while the incorrect dish was covered with a white lid. Both dishes contained food, but food in the incorrect dish was made inaccessible by wire mesh, thereby allowing us to control for any olfactory or vomeronasal cues that might reveal the correct dish. The position of the blue lid was randomized for each trial and counterbalanced across treatments. For each trial, we scored: (1) whether or not the lizard chose the correct (blue) dish; and (2) latency to access the food reward in the correct dish. Lizards were considered to have learnt the association task if they made the correct choice in $7 / 8$ consecutive trials. This is a relatively strict learning criterion, with the probability of getting 7 or more trials correct by chance out of 8 being 3\%, well below the $5 \%$ cut-off normally used. We gave the lizards a total of 26 trials to learn this task and all lizards learnt in this time. We decided on 26 trials somewhat subjectively prior to the experiment based on our experience with this species (Riley et al., 2017, 2018), and because we wanted to reduce the probability of reaching a 7/8 criterion simply by chance, which increases with a larger number of trials.

The visual acuity of tree skinks has not been studied previously, however, diurnal lizards have tetrachromatic vision and therefore, a good ability to discriminate different colors and brightness (Fleishman et al., 2011). Males and females are similarly colored and lizards have conserved visual systems (Fleishman et al., 2011), with no evidence for sexual effects on color preference. We independently tested for any color preference (to be sure that lizards did not prefer blue to white) using 12 individuals (10 males and 2 females) with the same history as our study animals. They were given a choice between a white vs. blue dish in which both contained an equal quantity of a food reward $\left(0.5 \mathrm{~g} \mathrm{Heinz}{ }^{\circledR}\right.$ fruit baby food). We conducted 10 trials over 5 days and randomized left vs. right locations to account for any side bias or spatial learning. A researcher from outside the study with no knowledge about lizards or this study scored and ran the experiment and recorded the color of the first dish a lizard ate from. During the first trial, skinks did not significantly differ in their choice between blue (chosen 8 times) 
and white (chosen 4 times) dishes (chi-square test: $\chi_{11}=0.69, P$ $=0.41)$. Across all trials and lizards $(N=119)$, there was also no significant difference in the number of choices between blue and white dishes (chi-square test: $\chi_{119}=0.02, P=0.90$ ). On average, the proportion of trials in which lizards selected the blue and white dish were $0.51 \pm 0.04$ (mean \pm standard error) and 0.49 \pm 0.04 , respectively, providing no evidence for a color preference between blue and white in E. striolata.

\section{Statistical Analyses}

All analyses were carried out in R ( $\mathrm{v}$ 3.3.0, R Development Core Team, 2010). In all models, treatment (social learning and control) and batch identity (batch one and two) were coded as two level factors. Batch identity was included in all models as a covariate. Before analyses, we first considered potential motivational differences between treatment groups. All observer lizards met our threshold of attempting at least $80 \%$ of tasks, thus motivation should not have confounded our results. Data for this study can be accessed at (Figshare: doi: 10.6084/m9.figshare.4981958).

We first examined if there were differences in learning speed and number of errors made during the learning phase (i.e., prior to meeting the learning criterion) between treatments using generalized linear models (GLM) from the R package "MASS" (Ripley et al., 2013). We modeled the mean trials taken to reach the learning criterion for each task, as well as the number of errors during the learning phase for the association task with a negative binomial error distribution.

We then asked whether the proportion of lizards that learnt in each trial for both tasks differed between treatments using a Cox regression proportional hazard survival analysis from the "survival" R package (Therneau and Lumley, 2016). Lizards that did not learn the task were considered as "right-censored" ( $n=2$ in the instrumental task).

We also tested if the probability of making the correct choice with each successive trial differed between treatments. We used Bayesian generalized linear mixed effects models (GLMM) from the "MCMCglmm" R package (Hadfield, 2010) to analyze (1) the probability of making a correct choice; and (2) the probability of removing the correct lid only (i.e., not removing the incorrect lid after removing the correct one) with a binomial error distribution (logit link). Whether an observer displaces one or both lids can inform about the potential mechanisms of learning (Noble et al., 2014). A mixed modeling framework was used to account for repeated measurements of the same individuals over time. We included an interaction term between treatment and trial, and modeled the change in both response variables between the treatment groups across trials. Default uniform priors were used for fixed effects. The residual variance was fixed at one, as this is what the "logit" family assumes (Hadfield, 2010). Lizard identity was fitted as a random intercept and trial number as a random slope. We specified the prior variance-covariance matrix of the random effects as $\mathrm{V}=\left[\begin{array}{ll}1 & 0 \\ 0 & 1\end{array}\right]$ with $\mathrm{nu}=0.002$. We ran three independent chains of 110,000 iterations, with a thinning interval of 100 and a burn-in of 10,000. The Gelman-Rubin test in the R package "coda" was used to ensure the three chains converged (Plummer et al., 2006). The trace plots of all chains were visually inspected to ensure samples were mixing well. Autocorrelation of the chains was assessed to ensure levels were low (lag $<0.1$ ) using the "autocorr" function, and we also performed Geweke and Heidelberg diagnostics (also using the R package "coda"). Pooled posterior modes from the three chains and 95\% credible intervals are reported, and parameter estimates were considered significant when the credible intervals did not include 0 . If interaction terms were not significant, they were removed and the models were rerun, thus, in this case, main effect estimates are derived from models with non-significant interaction terms excluded.

\section{RESULTS}

\section{Instrumental Task}

Twenty-six of 28 lizards (93\%) met our learning criterion including all 15 (100\%) lizards from the social learning treatment and $11 / 13(84 \%)$ from the control. The proportion of lizards that learnt the task for each trial did not differ between treatments (estimate $=0.50, \mathrm{SE}=0.41, P=0.23$; Figure 2A). The mean number of trials required to reach our learning criterion also did not differ significantly between the social learning treatment and control (Figure 2B, Table 1A).

\section{Association Task}

All lizards (100\%) from both treatments reached the learning criterion for the association task. We assessed whether lizards retained a significant binomial choice over at least 8 trials (or more) after reaching learning criterion (i.e., continuing to choose the correct dish): 17/27 (63\%) of individuals retained a significant tally, while the remainder showed extinction of this choice (see section Discussion). The proportion of lizards that learnt was not significantly different between the two groups (estimate $=0.75$, $\mathrm{SE}=0.46, P=0.10$; Figure 3A).

Social learners learnt the task, on average, in significantly fewer trials compared to the control (Figure 3B, Table 1B). Moreover, social learners made, on average, significantly fewer errors compared to the control during the learning stage (Figure 3C, Table 2). Social learners also had a significantly higher probability of making a correct choice (Table 3A). Interestingly, we found a significant treatment by trial interaction effect for the probability of making a correct choice (Table 3A). Social learners had a much higher intercept for the probability of making a correct choice compared to control lizards (Figure 3D). However, as trials progressed, the probability of making a correct choice for social learners decreased, while it slowly increased for control lizards (Figure 3D). In contrast, when we examined the probability of removing the correct lid only (as opposed to both lids), there was no treatment effect and the interaction between treatment and trial was not significant (Table 3B).

\section{DISCUSSION}

Only two of 28 lizards (control group) did not reach criterion in the instrumental task and the mean number of trials required to reach criterion was not significantly influenced by treatment (social learning vs. control) in the remaining 26 lizards. This is probably because the instrumental task is relatively simple and 

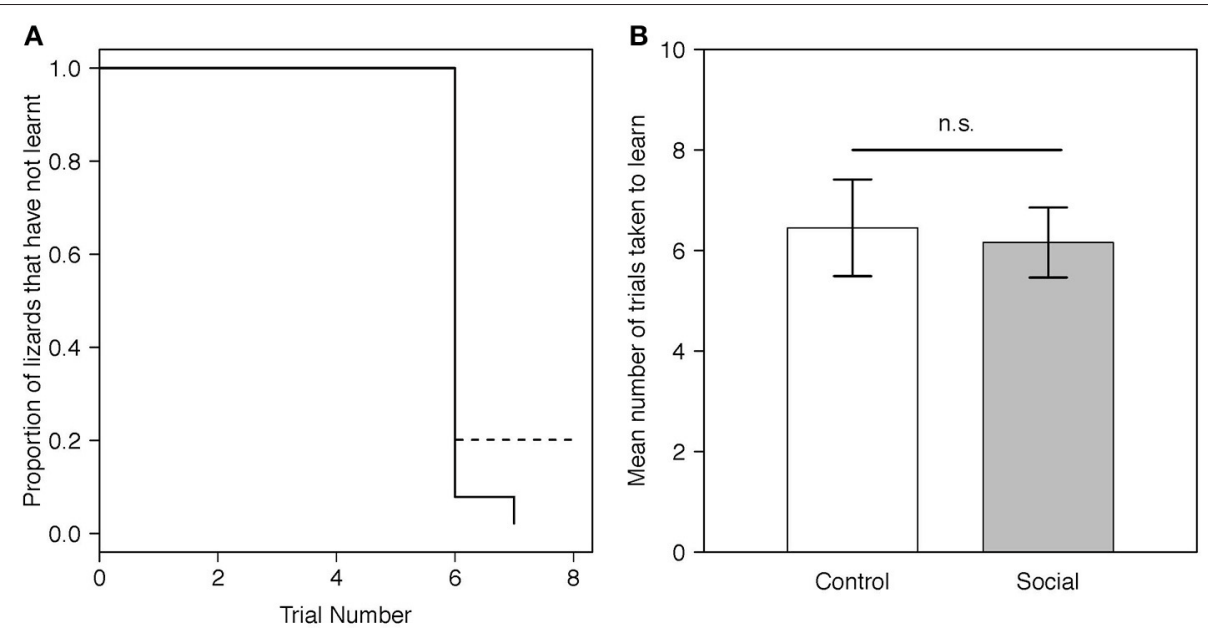

FIGURE 2 | (A) Predicted proportion of lizards across trials in the social learning treatment $(n=15)$ and control group $(n=13)$ that did not learn the instrumental task. Predictions were averaged across both batches. The solid line represents the social learning treatment, the dashed line the control group. (B) Predicted mean number of trials taken to learn for lizards in the social learning treatment $(n=15)$ and control group $(n=13)$ in the instrumental task (Table 1A). For visualization purposes, batch number was set to 1. Gray bars represent the social learning treatment; white bars represent the control group. Error bars are one standard error.

TABLE 1 | Estimates and standard errors (SE) from a generalized linear model (GLM) examining the effects of a lizard's treatment group (social learning or control) on the mean number of trials it took for a lizard to learn the $(\mathbf{A})$ instrumental task $(N=28)$, (B) association task $(N=28)$.

\begin{tabular}{lccccc}
\hline & \multicolumn{2}{c}{$(\mathbf{A})$ Instrumental task } & & \multicolumn{2}{c}{ (B) Association task } \\
\cline { 2 - 3 } \cline { 6 - 6 } & Estimate & SE & & Estimate & SE \\
\hline Intercept & 1.92 & 0.29 & & 2.42 & 0.12 \\
Treatment (Social) & -0.05 & 0.16 & & $-\mathbf{0 . 3}$ & $\mathbf{0 . 1 3}$ \\
Batch (Batch 2) & -0.06 & 0.17 & & $\mathbf{0 . 2 8}$ & $\mathbf{0 . 1 3}$ \\
\hline
\end{tabular}

Bolded estimates are significant. Baseline is the control treatment, and batch 1.

lizards were able to solve it rapidly, even in the absence of social demonstrators. All 28 lizards reached criterion in the association task where there was a significant treatment effect: lizards in the social learning treatment made fewer errors and reached criterion in significantly fewer trials than lizards in the control group (individual learning).

We found that adult female E. striolata can use social information from other adult females to learn foraging tasks. This is in contrast to a previous study of social learning in the same species (E. striolata), where there was no evidence that social information was used by juveniles reared under two different social environments to learn a similar foraging-based task (Riley et al., 2018). The benefits and use of social information can depend strongly on an individual's age, and the environment and social context in which animals develop or reside (Laland, 2004; Noble et al., 2014; Kar et al., 2017; Riley et al., 2018). The lack of social information use in juvenile E. striolata may be due to their age, or, alternatively, the social context in which the learning tasks were presented (Riley et al., 2018). Unlike our study, juveniles observed unfamiliar adult female demonstrators which can be lethally aggressive to juveniles in the wild (Chapple,
2003; O'Connor and Shine, 2004). Thus, juveniles may have not been motivated to visit a location in which the female was present in order to avoid aggressive interactions. Likewise, young guppies (Poecilia reticulata) do not socially learn from adults until they are large enough not be negatively impacted by aggressive interactions (Leris and Reader, 2016).

Interestingly, once lizards had reached criterion, we found that the probability of making a correct choice decreased over time (trial number) for the social group while it steadily increased in the control group. Also, of the lizards that showed extinction of learning, $60 \%(6 / 10)$ were from the social learning treatment. Social learning can be strategic and may change in response to changes in the environment. In our study, social information from the demonstrator about the location of food may be beneficial initially, but over time, as an observer sees the same individual feed from the same location, they also receive information about potential competition (with the demonstrator) and resource depletion. Therefore, it may be beneficial for an observer to switch to an individual learning strategy and forage elsewhere, in this case by sampling the incorrect dish more often. A similar strategy occurs in threespined sticklebacks (Gasterosteus aculeatus) in relation to food patch availability. Sticklebacks rely on social information when food patches are large and adopt individual-specific foraging tactics when patches are small (Hansen et al., 2016). Furthermore, the value of a constant food resource may decline with time, particularly given the need for nutrient regulation where diet diversity is favored over quantity in many species (Simpson et al., 2004). Our results therefore suggest a role for social feedback in informing individual learning strategies, which has been demonstrated in many other systems (Hoppitt and Laland, 2013).

Social learning is governed by multiple mechanisms (reviewed in Whiten and Ham, 1992; Zentall, 2011; Hoppitt and Laland, 2013) including local/stimulus enhancement and 
A

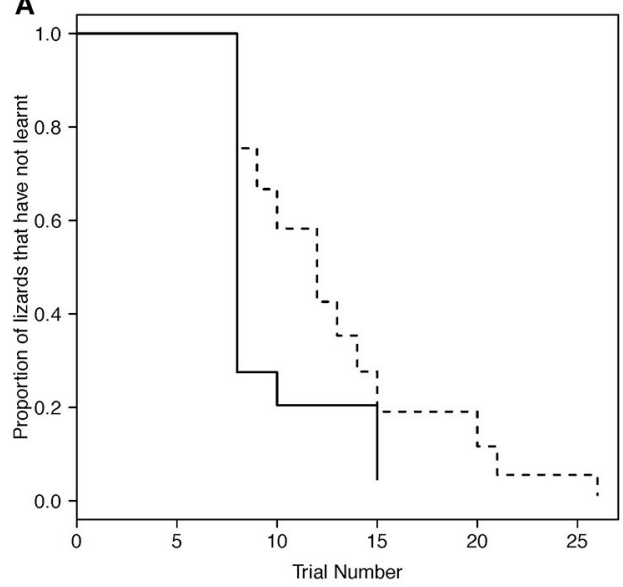

C

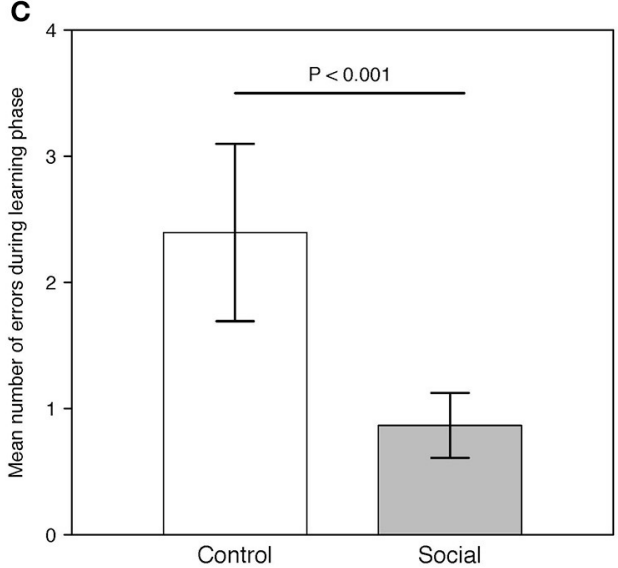

B

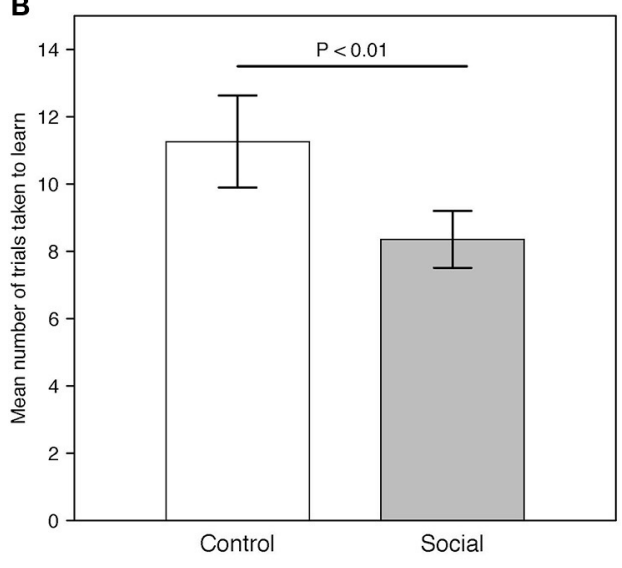

D

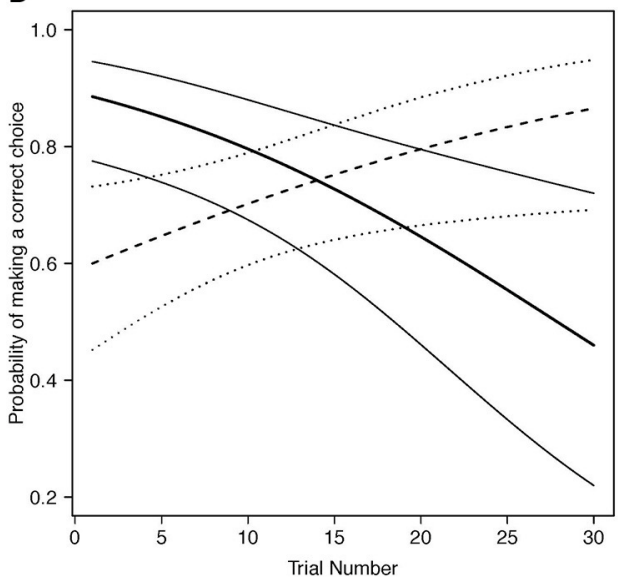

FIGURE 3 | (A) Predicted proportion of lizards across trials in the social learning treatment $(n=15)$ and control group $(n=13)$ that did not learn the association task. Predictions were averaged across both batches. The solid line represents the social learning treatment, the dashed line the control group. (B) Predicted mean number of trials taken to learn for lizards in the social learning treatment $(n=15)$ and control group $(n=13)$ in the association task (Table 1B). (C) Predicted mean number of errors made by lizards during the learning phase (i.e., prior to reaching the learning criterion) in the social learning treatment and control group in the association task (Table 2). For visualization purposes, batch number was set to 1. Gray bars represent the social learning treatment; white bars represent the control group. Error bars represent the standard error. (D) Predicted probability of making a correct choice across trials for lizards in the social learning treatment $(n=15)$ and control group $(n$ $=13$ ) in the association task (Table 3A). For visualization purposes, batch number was set to 1 . The thick solid line represents the social learning treatment and the thick dashed line the control group. Thin solid lines and the dotted lines represent the $95 \%$ credible interval for the social learning treatment and the control group, respectively.

TABLE 2 | Estimates and standard errors (SE) from a generalized linear model (GLM) examining the effects of a lizard's treatment group (social learning or control) on the mean number of errors made by lizards during the learning phase in the association task $(N=28)$.

\begin{tabular}{lcc}
\hline & Estimate & SE \\
\hline Intercept & 0.87 & 0.29 \\
Treatment (Social) & $\mathbf{- 1 . 0 2}$ & $\mathbf{0 . 3 4}$ \\
Batch (Batch 2) & $\mathbf{0 . 6 7}$ & $\mathbf{0 . 3 2}$
\end{tabular}

Bolded estimates are significant. Baseline is the control treatment, and batch 1.

imitation/copying (Hoppitt and Laland, 2013; Kis et al., 2015). While we did not design a task to specifically test for social learning mechanisms, we predicted that once lizards learnt the correct dish, if they were using imitation mechanisms they would displace the lid from only the correct, food-containing dish, and not the incorrect dish. Given that the demonstrator could only displace the lid covering the dish with the reward, observers only saw the correct lid being removed. In the lizard E. quoyii, young, but not old, males will displace only the lid covering the reward dish once they have learnt the correct association (Noble et al., 2014), which is suggestive of imitation. However, we found no significant difference in the propensity of lizards from the social learning and control group to remove the additional, incorrect lid. While we found no evidence of imitation using this design, additional cognitive testing is required to elucidate the mechanisms governing social learning in this species.

Kin selection has been proposed as a key factor influencing social learning because of the benefits of helping close relatives 
TABLE 3 | Pooled posterior modes and 95\% credible intervals from a Bayesian Markov chain Monte Carlo generalized linear mixed effects model (GLMM) examining the effects of (A) a lizard's treatment group (social learning or control), batch (1 or 2), and trial number on the log odds of making a correct choice, and (B) the log odds of choosing the correct dish only ( $\mathrm{N}_{\text {lizards }}=28, \mathrm{~N}_{\mathrm{obs}}=758$ ).

\begin{tabular}{|c|c|c|c|c|c|c|}
\hline & \multicolumn{3}{|c|}{ (A) Log odds of making of a correct choice } & \multicolumn{3}{|c|}{ (B) Log odds of removing the correct lid only } \\
\hline & Estimate & $\mathbf{L}$ & $\mathbf{U}$ & Estimate & $\mathbf{L}$ & $\mathbf{U}$ \\
\hline Intercept & 1.12 & 0.58 & 2.06 & -1.28 & -2.16 & -0.1 \\
\hline Treatment (Social) & 1.77 & 0.88 & 2.77 & -0.03 & -1.02 & 0.87 \\
\hline Trial & 0.05 & 0.01 & 0.1 & -0.03 & -0.09 & 0.01 \\
\hline Batch (Batch 2) & -0.76 & -1.39 & -0.1 & -3.75 & -5.41 & -2.37 \\
\hline Treatment $($ Social) $\times$ Trial & -0.13 & -0.2 & -0.06 & -0.04 & -0.13 & 0.07 \\
\hline
\end{tabular}

Bolded estimates are significant. Main effects are presented from a model without the interaction, when the interaction was not significant. Baseline is the control treatment, and batch 1. $L$ and $U$ represent the lower and upper credible intervals, respectively.

(Laland, 2004; Hoppitt and Laland, 2013). In many cases of social learning, an observer acts as a scrounger by using information obtained through observation (Barta and Giraldeau, 1998; Hoppitt and Laland, 2013). In other words, a "demonstrator" is not performing a behavior to directly assist an observer, but their actions provide useful information and because they are related an observer may be more inclined to act on this information (Laland, 2004). In family living lizards, such as the Egernia group, offspring can potentially use social information from their parents or siblings. Unfortunately, we were unable to control for relatedness in this study but were still able to detect social learning. It would be interesting to test for different rates of social learning among known siblings, and between parents and offspring, including both within males and between sexes.

In summary, female tree skinks use social information to solve a foraging task in the laboratory. To the best of our knowledge, this is the first demonstration of social learning in a family living lizard and adds to the evidence that social learning is prevalent in lizards (Noble et al., 2014; Kis et al., 2015; Pérez-Cembranos and Pérez-Mellado, 2015). More tests of social learning from systems with different evolutionary history, social systems, life history tactics, and ecological constraints, would be informative.

\section{REFERENCES}

Alem, S., Perry, C. J., Zhu, X., Loukola, O. J., Ingraham, T., Søvik, E., et al. (2016). Associative mechanisms allow for social learning and cultural transmission of string pulling in an insect. PLoS Biol. 14:e1002564. doi: 10.1371/journal.pbio.1002564

Barta, Z., and Giraldeau, L.-A. (1998). The effect of dominance hierarchy on the use of alternative foraging tactics: a phenotype-limited producing-scrounging game. Behav. Ecol. Sociobiol. 42, 217-223. doi: 10.1007/s002650050433

Boyd, R., and Richerson, P. J. (1985). Culture and the Evolutionary Process. Chicago, IL: University of Chicago Press.

Byrne, R. W. (2015). "Primate intelligence," Handbook of Paleoanthropology, 2nd Edn., eds W. Henke and I. Tattersall (Heidelberg: Springer-Verlag), 1721-1740.

Chapple, D. G. (2003). Ecology, life-history, and behavior in the Australian Scincid genus Egernia, with comments on the evolution of complex sociality in lizards. Herpetol. Monog. 17, 145-180. doi: 10.1655/07331347(2003)017[0145:ELABIT]2.0.CO;2

\section{AUTHOR CONTRIBUTIONS}

MW, FX, RB, and DN conceived and designed the experiments. FX collected the data. JR and FK analyzed the data with DN. All authors contributed to writing and editing of the manuscript.

\section{ACKNOWLEDGMENTS}

We thank Hamsini Bijlani for her assistance with laboratory color preference assays. This work was funded by the Australian Research Council (DP130102998 to MW and RB). DWAN was supported by an ARC DECRA (DE150101774) and UNSW VC Fellowship. JR was supported by the Natural Sciences and Engineering Research Council of Canada, and Macquarie University. All experimental procedures were approved by the Macquarie University Animal Ethics Committee (ARA2013/039) and followed the ASAB/ABS Guidelines for the Use of Animals in Research. Permission to conduct this research was granted by the NSW Office of Environment and Heritage (SL101264). Following the study, all lizards were transferred to our captive colony. We thank Thierry Bernard and Anindita Bhadra for their helpful comments that improved the quality of this ms.

Davis, K. M., and Burghardt, G. M. (2011). Turtles (Pseudemys nelsoni) learn about visual cues indicating food from experienced turtles. J. Comp. Psychol. 125, 404-410. doi: 10.1037/a0024784

Duckett, P. E., Morgan, M. H., and Stow, A. J. (2012). Tree-dwelling populations of the skink Egernia striolata aggregate in groups of close kin. Copeia 2012, 130-134. doi: 10.1643/CE-10-183

Enquist, M., Eriksson, K., and Ghirlanda, S. (2007). Critical social learning: a solution to rogers's paradox of nonadaptive culture. Am. Anthropol. 109, 727-734. doi: 10.1525/aa.2007.109.4.727

Fleishman, L. J., Loew, E. R., and Whiting, M. J. (2011). High sensitivity to short wavelengths in a lizard and implications for understanding the evolution of visual systems in lizards. Proc. R. Soc. B Biol. Sci. 278, 2891-2899. doi: 10.1098/rspb. 2011.0118

Giraldeau, L. A., and Beauchamp, G. (1999). Food exploitation: searching for the optimal joining policy. Trends Ecol. Evol. 14, 102-106. doi: 10.1016/S0169-5347(98)01542-0 
Giraldeau, L. A., Valone, T. J., and Templeton, J. J. (2002). Potential disadvantages of using socially acquired information. Philos. Trans. R. Soc. Lond. B 357, 1559-1566. doi: 10.1098/rstb.2002.1065

Hadfield, J. D. (2010). MCMC methods for multi-response generalized linear mixed models: the MCMCglmm R package. J. Stat. Softw. 33, 1-22. doi: $10.18637 /$ jss.v033.i02

Hamilton, W. D. (1964). The genetical evolution of social behaviour: I. J. Theor. Biol. 7, 1-16. doi: 10.1016/0022-5193(64)90038-4

Hansen, M. J., Ward, A. J. W., Fürtbauer, I., and King, A. J. (2016). Environmental quality determines finder-joiner dynamics in socially foraging three-spined sticklebacks (Gasterosteus aculeatus). Behav. Ecol. Sociobiol. 70, 889-899. doi: 10.1007/s00265-016-2111-5

Helfman, G. S., and Schultz, E. T. (1984). Social transmission of behavioral traditions in a coral reef fish. Anim. Behav. 32, 379-384. doi: 10.1016/S0003-3472(84)80272-9

Holzhaider, J. C., Hunt, G. R., and Gray, R. D. (2010). Social learning in new Caledonian crows. Learn. Behav. 38, 206-219. doi: 10.3758/LB.38.3.206

Hoppitt, W., and Laland, K. N. (2013). Social Learning: An Introduction to Mechanisms, Methods, and Models. Princeton, NJ: Princeton University Press.

Kar, F., Whiting, M. J., and Noble, D. W. A. (2017). Dominance and social information use in a lizard. Anim. Cogn. 20, 805-812. doi: 10.1007/s10071-017-1101-y

Kis, A., Huber, L., and Wilkinson, A. (2015). Social learning by imitation in a reptile (Pogona vitticeps). Anim. Cogn. 18, 325-331. doi: 10.1007/s10071-014-0803-7

Laland, K. N. (2004). Social learning strategies. Learn. Behav. 32, 4-14. doi: 10.3758/BF03196002

Leadbeater, E., and Chittka, L. (2007). Social learning in insects - from miniature brains to consensus building. Curr. Biol. 17, R703-R713. doi: 10.1016/j.cub.2007.06.012

Lefebvre, L. (1986). Cultural diffusion of a novel food-finding behavior in urban pigeons: an experimental field test. Ethology 71, 295-304. doi: 10.1111/j.1439-0310.1986.tb00594.x

Leris, I., and Reader, S. M. (2016). Age and early social environment influence guppy social learning propensities. Anim. Behav. 120, 11-19. doi: 10.1016/j.anbehav.2016.07.012

Liker, A., and Bartas, Z. (2002). The effects of dominance on social foraging tactic use in house sparrows. Behaviour 139, 1061-10761. doi: 10.1163/15685390260337903

Noble, D. W. A., Byrne, R. W., and Whiting, M. J. (2014). Age-dependent social learning in a lizard. Biol. Lett. 10:20140430. doi: 10.1098/rsbl. 2014.0430

O'Connor, D. E., and Shine, R. (2004). Parental care protects against infanticide in the lizard Egernia saxatilis (Scincidae). Anim. Behav. 68, 1361-1369. doi: 10.1016/j.anbehav.2004.02.014

Pérez-Cembranos, A., and Pérez-Mellado, V. (2015). Local enhancement and social foraging in a non-social insular lizard. Anim. Cogn. 18, 629-637. doi: 10.1007/s10071-014-0831-3

Plummer, M., Best, N., Cowles, K., and Vines, K. (2006). CODA: Convergence diagnosis and output analysis for MCMC. R News 6, 7-11.

R Development Core Team (2010). R: A Language and Environment for Statistical Computing. Vienna: R Foundation for Statistical Computing.

Reader, S. M. (2000). Social Learning and Innovation: Individual Differences, Diffusion Dynamics and Evolutionary Issues. Cambridge, UK: University of Cambridge.

Reader, S. M., Kendal, J. R., and Laland, K. N. (2003). Social learning of foraging sites and escape routes in wild Trinidadian guppies. Anim. Behav. 66, 729-739. doi: $10.1006 /$ anbe. 2003.2252
Reader, S. M., and Laland, K. N. (2002). Social intelligence, innovation, and enhanced brain size in primates. Proc. Natl. Acad. Sci.U.S.A. 99, 4436-4441. doi: 10.1073/pnas.062041299

Riley, J. L., KüChler, A., Damasio, T., Noble, D. W. A., Byrne, R. W., and Whiting, M. J. (2018). Learning ability is unaffected by isolation rearing in a family-living lizard. Behav. Ecol. Sociobiol. 72:20. doi: 10.1007/s00265-017-2435-9

Riley, J. L., Noble, D. W. A., Byrne, R. W., and Whiting, M. J. (2017). Does social environment influence learning ability in a family-living lizard? Anim. Cogn. 20, 449-458. doi: 10.1007/s10071-016-1068-0

Ripley, B., Venables, B., Bates, D. M., Hornik, K., Gebhardt, A., Firth, D., et al. (2013). Package 'MASS'. CRAN Repository. Oxford.

Simpson, S. J., Sibly, R. M., Lee, K. P., Behmer, S. T., and Raubenheimer, D. (2004). Optimal foraging when regulating intake of multiple nutrients. Anim. Behav. 68, 1299-1311. doi: 10.1016/j.anbehav.2004.03.003

Therneau, T. M., and Lumley, T. (2016). Package 'survival'. Verze.

Thonhauser, K. E., Gutnick, T., Byrne, R. A., Kral, K., Burghardt, G. M., and Kuba, M. J. (2013). Social learning in Cartilaginous fish (stingrays Potamotrygon falkneri). Anim. Cogn. 16, 927-932. doi: 10.1007/s10071-013-0625-Z

Thornton, A., and Clutton-Brock, T. (2011). Social learning and the development of individual and group behaviour in mammal societies. Philos. Trans. R. Soc. B Biol. Sci. 366, 978-987. doi: 10.1098/rstb.2010.0312

Thornton, A., and Malapert, A. (2009). Experimental evidence for social transmission of food acquisition techniques in wild meerkats. Anim. Behav. 78, 255-264. doi: 10.1016/j.anbehav.2009.04.021

While, G. M., Chapple, D. G., Gardner, M. G., Uller, T., and Whiting, M. J. (2015). Egernia lizards. Curr. Biol. 25, R593-R595. doi: 10.1016/j.cub.2015.02.070

Whiten, A. (2000). Primate culture and social learning. Cogn. Sci. 24, 477-508. doi: $10.1207 /$ s15516709cog2403_6

Whiten, A. (2011). The scope of culture in chimpanzees, humans and ancestral apes. Philos. Trans. R. Soc. Londn B 366, 997-1007. doi: 10.1098/rstb.2010.0334

Whiten, A., and Ham, R. (1992). "On the nature and evolution of imitation in the anim al kingdom: reappraisal of a century of research," in Advances in the Study of Behavior, eds P. J. B. Slater, J. S. Rosenblatt, C. Beer and M. Milinski (New York, NY: Academic Press), 239-283.

Whiting, M. J., and While, G. M. (2017). "Sociality in lizards," in Comparative Social Evolution, eds D. R. Rubenstein and P. Abbott (Cambridge: Cambridge University Press), 390-426.

Wilkinson, A., Kuenstner, K., Mueller, J., and Huber, L. (2010). Social learning in a nonsocial reptile (Geochelone carbonaria). Biol. Lett. 6, 614-616. doi: $10.1098 / \mathrm{rsbl} .2010 .0092$

Zentall, T. R. (2011). Social learning mechanisms: implications for a cognitive theory of imitation. Interact. Stud. Soc. Behav. Commun. Biol. Artif. Syst. 12, 233-261. doi: 10.1075/is.12.2.03zen

Conflict of Interest Statement: The authors declare that the research was conducted in the absence of any commercial or financial relationships that could be construed as a potential conflict of interest.

The reviewer, TB, and handling Editor declared their shared affiliation.

Copyright (C) 2018 Whiting, Xu, Kar, Riley, Byrne and Noble. This is an open-access article distributed under the terms of the Creative Commons Attribution License (CC $B Y)$. The use, distribution or reproduction in other forums is permitted, provided the original author(s) and the copyright owner are credited and that the original publication in this journal is cited, in accordance with accepted academic practice. No use, distribution or reproduction is permitted which does not comply with these terms. 